\title{
A SUCCESSFUL MANAGEMENT OF DENGUE FEVER IN PREGNANCY: REPORT OF TWO CASES
}

In the most recent outbreak of degue fever in India, we encountered at least seven cases of fever with thrombocytopenia in pregnancy but only two were seropositive for dengue. In one of the cases there was postpartum hemorrhage while in the other case there was perinatal transmission to the neonate requiring platelet transfusions. The diagnostic difficulties in pregnancy and the management are discussed.

Key Words: Dengue fever, pregnancy, vertical transmission

Dengue fever is a febrile disease found in the tropics. It is caused by one of the four closely related virus serotypes of the genus flavivirus, family flaviviridae, group IV ss RNA.
Dengue is transmitted to humans by the mosquito Aedes aegptii. Epidemic dengue has become more common since the 1980s and by the late 1990s dengue was the most important mosquito-borne disease affecting humans after malaria.

Significant outbreaks of dengue fever occur every five or six years. There tend to remain a large number of susceptible people in the population despite previous outbreaks because there are four different strains of the dengue virus and because of new susceptible individuals entering the target population, either through childbirth or immigration.

Dengue infection in pregnancy carries the risk of 
hemorrhage for both the mother and the newborn. In addition, there is a serious risk of premature birth and foetal death. In case of infection close to term, there is a risk of vertical transmission. Hence the knowledge of its diagnosis and management is of vital importance.

During a recent outbreak we encountered at least seven cases of fever with thrombocytopenia in pregnancy but only two were seropositive for dengue. We report here two interesting cases of pregnancy with dengue that came to our hospital and were managed successfully.

\section{Case Reports}

\section{Case 1}

The first case was unbooked, 20 years old, second gravida at 39 weeks gestation who presented with high grade fever $\left(102^{\circ} \mathrm{F}\right)$ with chills for one week associated with malaise and epigastric pain. There were no bleeding manifestations. At the time of examination, she was afebrile with stable vital signs. Blood pressure was 150/104. Urine protein was measured as trace. The laboratory investigations showed haemoglobin of $8.7 \mathrm{~g} / \mathrm{dL}$, haematocrit of $35 \%$, a total leukocyte count of $8,200 / \mathrm{mm}^{3}$ and a platelet count of $10,000 / \mathrm{mm}^{3}$.

In view of the ongoing dengue outbreak, a possibility of dengue fever was considered along with the other common differentials of thrombocytopenia with term pregnancy with preeclampsia being the primary diagnosis. Induction for term pregnancy with preeclampsia was deferred in view of the low platelets counts.

Her dengue serology was positive (IgM and IgG). Her liver and renal function tests were well within limits indicating that the thrombocytopenia was indeed due to dengue and not a part of the preeclamptic toxaemia.

She received six units of random donor platelets. Post transfusion her platelets were around $1,00,000 / \mathrm{mm}^{3}$. Within 24 hours she went into spontaneous labour and delivered a healthy $2.6 \mathrm{~kg}$ male baby. The delivery was uncomplicated but twelve hours after delivery she had postpartum hemorrhage. Blood loss was approximately $400 \mathrm{~mL}$. She responded to uterotonics and uterine massage. Repeat platelet count after delivery was $96,000 / \mathrm{mm}^{3}$. There were no further bleeding episodes. Mother and the infant were discharged in a stable condition. As the infant was asymptomatic, serological screening was not done for dengue.

\section{Case 2}

The second case was a 27-year-old primigravida, with gestational hypertension without proteinuria diagnosed at 22 weeks gestation controlled on methyldopa $250 \mathrm{mg}$ thrice daily. At 39 weeks period of gestation she presented with high-grade fever for one day and decreased foetal movements. On examination, her general condition was stable with a temperature of $102^{\circ} \mathrm{F}$. Biophysical profile was $4 / 8$ with poor fetal tone, breathing and absent gross body movements. Necessary investigations for fever including complete haemogram and peripheral smear for malarial parasites, urine routine microscopy and cultures and blood cultures were done. Labour was induced with dinoprostone gel. Emergency caesarean section was done for meconium stained liquor and late decelerations in foetal heart rate during labour. Postoperatively she developed fever on second day and her platelet counts showed a falling trend to up to $22,000 \mathrm{~mm}^{3}$ on the fourth postoperative day. Her dengue serology was positive for both $\operatorname{IgG}$ and $\operatorname{IgM}$. She received one unit of single donor platelet and 11 units of random donor platelets and intravenous antibiotics for 7 days. She was afebrile after seventh postoperative day and her platelet count was $1,68,000 / \mathrm{mm}^{3}$.

The baby was admitted in NICU on third day of life with fever, cold extremities, erythematous rash and hepatosplenomegaly. A differential diagnosis of sepsis or dengue was considered. In view of thrombocytopenia, $50 \mathrm{~mL}$ of platelets was transfused and the baby received intravenous fluids and intravenous antibiotics. Dengue serology was IgM positive and RT-PCR was positive for dengue type II. For RT-PCR, viral RNA was extracted from the serum sample using the automated MAGNA Pure compact nucleic acid isolation system (Roche, Switzerland) as per manufacturers' instructions. Extracted RNA was stored at $-70^{\circ} \mathrm{C}$ till it was used for RT-PCR. Published primers by Lanciotti et al were used. ${ }^{[4]}$ The viral RNA was reverse transcribed to cDNA using avian myeloblastosis virus reverse transcriptase enzyme (AMV RT), (Promega Corp., USA) and the dengue virus downstream consensus primer (D2), common to all four dengue serotypes. Briefly, for each $25 \mu \mathrm{L}$ reaction, $10 \mu \mathrm{L}$ RNA and $50 \mathrm{pM}$ of primer DENV-2 along with $200 \mu \mathrm{M}$ of each deoxynucleoside triphosphate (dNTP) (Promega Corp., USA) and 20 units of AMV RT were used. The samples were incubated for 90 minutes at $42^{\circ} \mathrm{C}$ followed by heating at $70^{\circ} \mathrm{C}$ for 15 minutes to inactivate the enzyme. For DNA amplification, $3 \mu \mathrm{L}$ of first-strand cDNA reaction was amplified in a total volume of $25 \mu \mathrm{L}$ containing $20 \mathrm{pM}$ of each forward and reverse primer, $200 \mu \mathrm{M}$ each of the four dNTPs (Promega, Madison, WI), 3 units of the Taq polymerase (Bangalore Genei, India) and $1.5 \mathrm{mM} \mathrm{MgCl} 2$. The reactions were allowed to proceed in a thermocycler gene Amp PCR System 9700 (ABI, USA). Dengue virus typing was done by second-round amplification with type-specific primers. Thermal cycling was performed and specific band size for each dengue virus serotypes observed. ${ }^{[4]} \mathrm{PCR}$ products were visualized under a digital gel documentation system (Bio-Rad, UK).

The mother and baby were discharged on day-14 in stable condition. 


\section{Discussion}

A WHO definition of dengue haemorrhagic fever ${ }^{[1]}$ for diagnosis has been in use since 1975 which states that all four of the following criteria must be fulfilled: fever, haemorrhagic tendency, thrombocytopenia and evidence of plasma leak as evidenced by haematocrit $20 \%$ higher than expected or a drop in haematocrit of $20 \%$ or more from the baseline following IV fluid, pleural effusion or ascites. All the above along with a weak rapid pulse and a narrow pulse pressure or hypotension and cold clammy skin and restlessness constitutes the dengue shock syndrome.

Although our patients did not fulfill all the clinical and investigative criteria as defined by WHO, they had fever with thrombocytopenia. Neither of them had a high haematocrit value but the physiological changes of pregnancy have to be kept in mind before interpreting the laboratory findings. The normal haematocrit range in pregnant women may be as low as 34\% (normal for a non-pregnant woman is $37 \%-47 \%$ ). ${ }^{[2]}$ This discrepancy has been explained by the physiologic anemia of pregnancy.

Thrombocytopenia, observed in about $7 \%$ of pregnancies, may be related to previously acquired diseases of immunological destruction as in idiopathic thrombocytopanic purpura (ITP), systemic lupus erythematosus (SLE), and antiphospholipid antibody syndrome (APA syndrome) or inherited diseases or to pregnancy-related complications such as pre-eclampsia, sepsis, or disseminated intravascular coagulation in obstetrics cases ${ }^{[3]}$ In $75 \%$ of the cases, thrombocytopenia cannot be referred to any of these aetiologies. All these differential diagnoses have to be kept in mind in a pregnant patient with thrombocytopenia, more so in the second case with preeclampsia.

Serology and molecular studies are available to confirm the diagnosis of dengue. Both the cases were IgG and IgM positive for dengue. IgG is non-specific and cross reacts with other flaviviruses. However, IgM capture ELISA is a widely used, simple and rapid method to detect Dengue specific IgM antibodies by capturing them out of solution using antihuman IgM previously bound to the solid phase. ${ }^{[5]}$ Nearly all patients (93\%) develop detectable IgM antibody 6 to 10 days after onset, and $99 \%$ of patients tested between 10 and 20 days have detectable IgM antibody. A small percentage of patients with secondary infections have no detectable IgM antibody. ${ }^{[5]}$

RT-PCR or virus isolation tests were not performed on our patients but the epidemiological studies done during the outbreak indicated the possibility of a serotype 2 outbreak which has a propensity to cause dengue haemorrhagic fever with shock. This was confirmed by the RT-PCR of one of the babies showing dengue serotype 2 .
One patient needed platelet transfusion in view of the very low platelet counts. But even after transfusion she developed postpartum hemorrhage 12 hours after delivery. This emphasises the potential hazard of antepartum and postpartum hemorrhage in pregnant patients with dengue over and above the risk to the general population. It is recommended that platelets be maintained at least around 50 x 10(9)/L at near term and delivery.

Vertical transmission of dengue has been reported in several studies. ${ }^{[6]}$ In most of them, mothers had an uncomplicated antenatal period and developed fever within a week of delivery. Some of them had preeclampsia and thrombocytopenia due to HELLP syndrome which was kept as a differential diagnoses in one of our patients. ${ }^{[7]}$ In case of infection close to term, there is a risk of hemorrhage for both the mother and the newborn. ${ }^{[8]}$ They reported vertical transmission in two out of the 38 mothers with dengue, while a previous study from India of eight cases during an outbreak of dengue did not report any vertical transmission. ${ }^{[9]}$ We report here a case of vertical transmission. The consequences of dengue infection can be serious with even neonatal death being reported in some studies. ${ }^{[10]}$ Most neonates developed fever within four to five days of life and consequently developed thrombocytopenia requiring platelet transfusions as was also seen in our cases.

\section{References}

1. World Health Organization. Dengue Hemorrhagic feverDiagnosis, treatment, prevention and control. Geneva 1997. p. 5-7,34.

2. Cantagallo F, Perini M, Cantagallo AA. Evaluation of hemoglobin and hematocrit in pregnant women receiving folate and iron supplements. Minerva Ginecol 1997;49:571-6.

3. Kaplan C, Forestier F, Dreyfus M, Morel-Kopp MC, Tchernia G. Maternal thrombocytopenia during pregnancy: Diagnosis and etiology. Semin Thromb Hemost 1995;21:85.

4. Lanciotti RS, Calisher C, Gubler DJ, Chang G, Vorndam V. Rapid detection and typing of dengue viruses from clinical samples by using reverse transcriptase-polymerase chain reaction. J Clin Virol 1992;30:545-55.

5. Kuno G, Gomez I, Gubler DJ. An ELISA procedure for the diagnosis of dengue infections. J Virol Methods 1991;33:10113.

6. Chotigeat U, Kalayanrooj S, Nisalak A. Vertical transmission of dengue infection in Thai infants: Two case reports. J Med Assoc Thai 2003;86:S628-32.

7. Chve JK, Lim CT, Ng KB, Lim JM, George R, Lam SK. Vertical transmission of dengue. Clin Infect Dis 1997;25:1347-7.

8. Carles G, Peiffer H, Talarmin A. Dengue fever and pregnancy: A study of 38 cases in french Guiana. J Gynecol Obstet Biol Reprod (Paris) 2000;29:758-62.

9. Malhotra N, Chanana C, Kumar S. Dengue infection in pregnancy. Int J Gynaecol Obstet 2006;94:131-2.

10. Carles G, Peiffer H, Talarmin A. Effects of dengue fever during pregnancy in French Guiana. Clin Infect Dis 1999;28:637-40. 
N Singh, *KA Sharma, V Dadhwal, S Mittal, AS Selvi

Departments of Obstetrics and Gynaecology (NS, KAS, VD, SM); and Lab Medicine (ASS), All India Institute of Medical Sciences,
New Delhi - 110 029, India

*Corresponding author(email: <k_aparna1@rediffmail.com>) Received: 10-01-08

Accepted: 19-04-08 